\title{
0 impacto da desnutrição no desenvolvimento e na gravidade das úlceras por pressão: uma revisão da literatura
}

\author{
Letícia Pedroni ${ }^{\star}$, Simone Bonatto**, Karina Mendes***
}

\section{Resumo}

O objetivo deste estudo foi avaliar, a partir de evidências científicas, se existe relação entre desnutrição e o risco de úlcera por pressão, bem como estabelecer a relação da desnutrição com a gravidade dessa enfermidade. O método adotado foi uma revisão da literatura, na qual foi utilizada a base de dados online Scopus, utilizando os seguintes descritores: pressure ulcer and nutrition. A consulta à base de dados foi realizada no mês de agosto de 2013. Foram selecionadas publicações dos últimos cinco anos, nos idiomas inglês, português e espanhol. No total, oito artigos foram utilizados para essa revisão da literatura. Todos os estudos mostraram que o risco de desenvolver úlceras por pressão é maior em pacientes desnutridos. Quatro estudos mostraram o aumento da probabilidade de ter uma úlcera por pressão mais grave ou maior número de úlceras por pressão com o aumento da severidade da desnutrição. Assim, comprova-se que o estado nutricional deficitário tem um papel importante na gênese da úlcera por pressão e uma nítida relação com a sua gravidade. A avaliação nutricional é de suma importância, pois por meio dela os pacientes que apresentam risco para o desenvolvimento de úlceras por pressão podem ser identificados precocemente, evitando maiores transtornos e custos hospitalares. Com isso, a avaliação do estado nutricional tem papel fundamental na prevenção e/ou na recuperação dessa enfermidade. Contudo, apesar das evidências encontradas, são necessários futuros estudos, com maior rigidez metodológica para reforçar essa associação.

Palavras-chave: Úlceras por pressão. Estado nutricional. Desnutrição.

\section{Introdução}

As úlceras por pressão (UP) também conhecidas como úlceras de decúbito ou escaras consistem em uma injúria localizada na pele e no tecido subjacente, que usualmente ocorre sobre proeminências ósseas, como cotovelos, tornozelos e quadris, ou em áreas que recebem grande quantidade de pressão

* Acadêmica do curso de Nutrição da Universidade de Caxias do Sul (UCS), Caxias do Sul/RS. Endereço: Rua Juvenil Benetti, 43, Santa Catarina, CEP: 95030-500, Caxias do Sul - RS E-mail: leticia.pedroni@hotmail.com.

** Mestre em Saúde Coletiva pela Universidade do Vale do Rio dos Sinos. Professora do Centro de Ciências da Saúde da Universidade de Caxias do Sul.

*** Doutora em Endocrinologia pela Universidade Federal do Rio Grande do Sul. Mestre em Saúde Coletiva pela Universidade do Vale do Rio dos Sinos. Professora do Centro de Ciências da Saúde da Universidade de Caxias do Sul.

$\rightarrow$ http://dx.doi.org/10.5335/rbceh.2014.3891

Recebido em: 23/03/2014. Aprovado em: 10/06/2014 
(MEIJERS et al., 2008; NPUAP/EPUAP; 2009). A compressão do tecido sensível sobre a proeminência óssea resulta em subsequente redução do fluxo sanguíneo e isquemia do local, causando hipóxia dérmica, necrose, ruptura da epiderme e, eventualmente, contaminação bacteriana (COSTA, 2003; CUNHA et al., 2000).

As UP apresentam incidência e alta prevalência em pacientes hospitalizados, tanto em centros de cuidados primários, como em casos de cuidados terciários ou em instituições especializadas para idosos e deficientes físicos. Há uma relação direta entre a idade do paciente, as características da instituição em que esse se encontra com o surgimento das UP. Em unidades hospitalares de atendimento de urgência, a prevalência varia de $5 \%$ a $15 \%$, em casas de repouso, de $15 \%$ e $20 \%$ e, em centros de reabilitação, de $30 \%$ a $50 \%$ (PROJETO DIRETRIZES, 2011; BOURS et al., 2002).

Sua etiologia depende de fatores extrínsecos, como pressão, força de tração, força de fricção, maceração/umidade excessivas, desidratação, e, também está ligada a fatores intrínsecos, como imobilidade, alterações da sensibilidade, incontinência urinária ou fecal - que leva à umidade local-, alterações do estado de consciência, idade avançada, má perfusão/oxigenação tecidual, infecção, anemia, deterioração do estado nutricional, comorbidades relacionadas com gasto metabólico elevado, doenças crônicas, etc. (SERPA, 2008; OLIVEIRA et al., 2009; SILVA; OLIVEIRA; SILVEIRA, 2009).

O peso corpóreo tem sido apontado como um fator relacionado ao desenvolvimento e à gravidade das UP. O IMC abaixo de $18,5 \mathrm{~kg} / \mathrm{m}^{2}$ está associado à diminuição da gordura corporal e, por conseguinte, redução da proteção contra a pressão em áreas ósseas proeminentes. A desnutrição protéico-calórica grave altera a regeneração tissular, a reação inflamatória e a função imune, tornando os indivíduos mais vulneráveis ao desenvolvimento de UP. Em idosos, o baixo nível de albumina sérica pode ser considerado evidência de desnutrição protéica e hipercatabolismo (SERPA, 2008; PROJETO DIRETRIZES, 2011).

Várias escalas estão disponíveis para avaliar o risco de desenvolvimento de UP. A escala de Braden é considerada por muitos autores a mais eficaz e tem sido a mais adotada em nível internacional. Essa escala é composta por seis subescalas: percepção sensorial, umidade da pele, atividade, mobilidade, estado nutricional, fricção e cisalhamento $(\mathrm{T}$. A. ARAÚJO; M. F. M. ARAÚJO; CAETANO, 2012).

Os sistemas de classificação das UP existem com objetivo de facilitar a identificação da lesão a partir da descrição de suas características. A classificação do National Pressure Ulcer Advisory Panel (NPUAP) classifica as UP, segundo sua gravidade em: estágio I - eritema da pele intacta que não embranquece após a remoção da pressão; estágio II perda da pele envolvendo a epiderme, derme ou ambas, a úlcera é superficial e apresenta-se como abrasão ou cratera rasa; estágio III - perda da pele na sua espessura total, envolvendo danos ou necroses do tecido subcutâneo que pode se aprofundar, não chegando até a fáscia, a úlcera se apresenta clinicamente como 
cratera profunda e estágio IV - perda da pele na sua total espessura com extensa destruição, necrose dos tecidos ou danos aos músculos, ossos ou estruturas de suporte como tendões ou cápsula das articulações (NPUAP/EPUAP; 2009).

Considerando o estado nutricional como fator fundamental na etiopatogenia da UP e para melhor elucidar o tema, a presente revisão tem como objetivo avaliar a relação entre a desnutrição e o risco de úlcera por pressão e a relação da desnutrição com a gravidade dessa enfermidade.

\section{Metodologia}

A metodologia utilizada para a realização desse estudo baseou-se em uma revisão da literatura. Para esta pesquisa foi utilizada a base de dados on-line Scopus. A consulta foi realizada em agosto de 2013.

$\mathrm{Na}$ estratégia de busca, utilizaram-se os descritores pressure ulcer and nutrition e o filtro em relação ao idioma (inglês, espanhol e português). Foram recuperados 91 documentos. Foi realizada a leitura dos títulos e dos resumos, quando o título e o resumo não eram esclarecedores, era realizada a busca e a leitura do artigo, para evitar que estudos importantes não fossem utilizados na revisão. Foram estabelecidos alguns critérios de exclusão nessa primeira etapa, como o fato de o artigo ser de revisão, não estar adequado ao tema ou apresentar data de publicação há mais de cinco anos. Assim, nessa etapa, foram selecionados 12 resumos. Desses, um foi excluído, pois não estava acessível, em seguida, foi realizada a leitura na íntegra dos 11 artigos restantes. Desses, outros três artigos foram excluídos porque não se adequavam ao objetivo do estudo. Ao final, oito artigos foram selecionados.

\section{Resultados}

Na Tabela 1, encontram-se os resultados mais relevantes de cada um dos oito artigos, bem como: nome dos autores, método utilizado para definição do estado nutricional, tipo de estudo, tamanho amostral, data de publicação e local.

Dos oito artigos selecionados, dois estudos foram realizados no Brasil, dois no Japão, um na Espanha, um na Austrália, um na Alemanha e um nos Estados Unidos. Do total de artigos selecionados, cinco artigos utilizaram delineamento transversal, um estudo foi retrospectivo por meio de análise de prontuários, um estudo foi de caso-controle e um estudo foi prospectivo, em que os pacientes foram acompanhados por 12 meses. Os estudos contaram com uma amostra que variou de 100 até 6.460 pessoas. Quanto aos locais de realização dos estudos, quatro foram realizados em hospitais, dois em domicílios e dois foram efetivados em hospital e em casa geriátrica. A idade mínima verificada esteve entre a faixa etária dos 14 anos e a máxima de 104 anos.

Todos os estudos mostraram que o risco de desenvolver UP é maior em pacientes desnutridos. Quatro estudos mostraram o aumento da probabilidade de ter uma UP mais grave ou um maior número de UP com o aumento da severidade da desnutrição. 


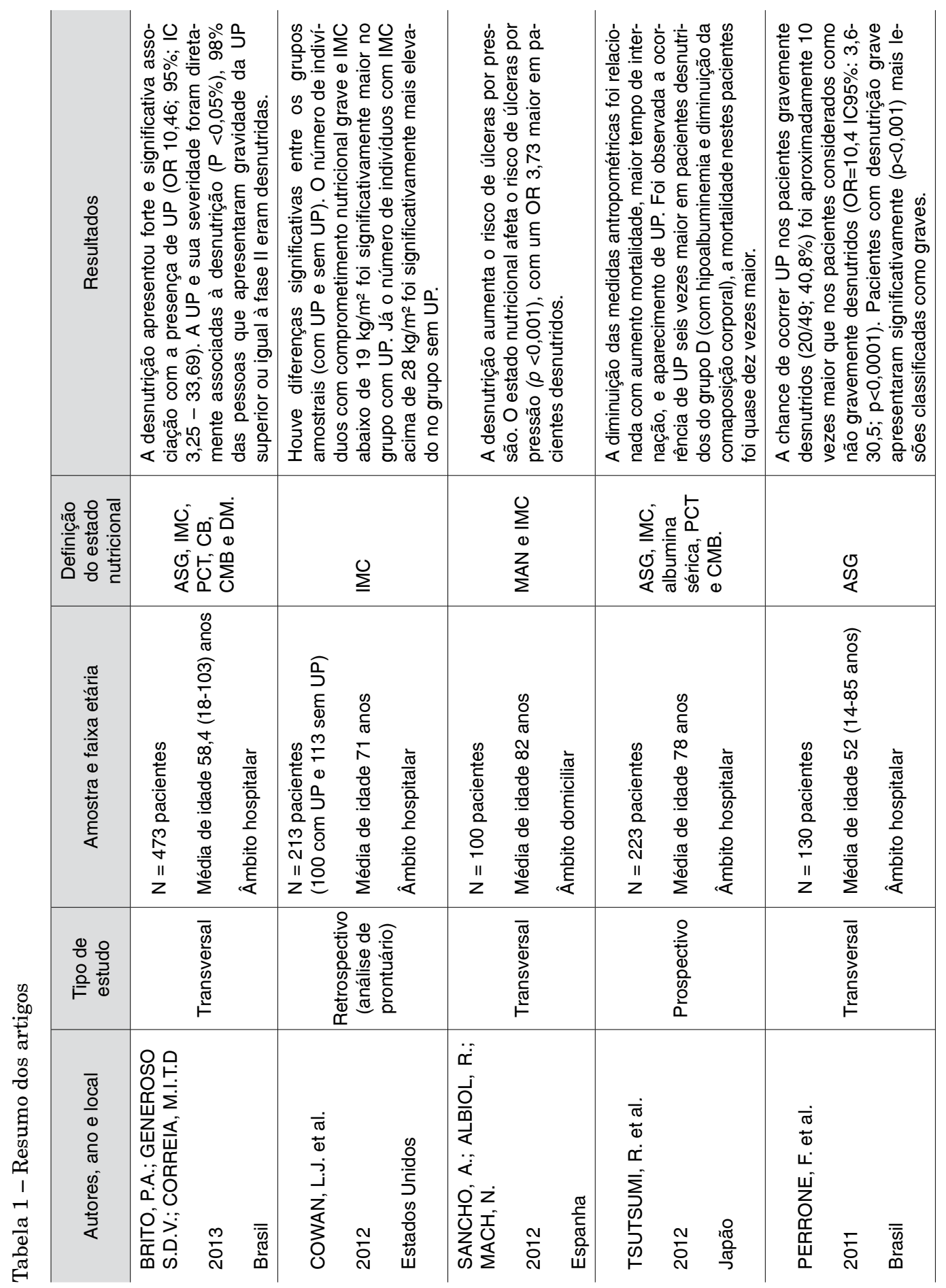




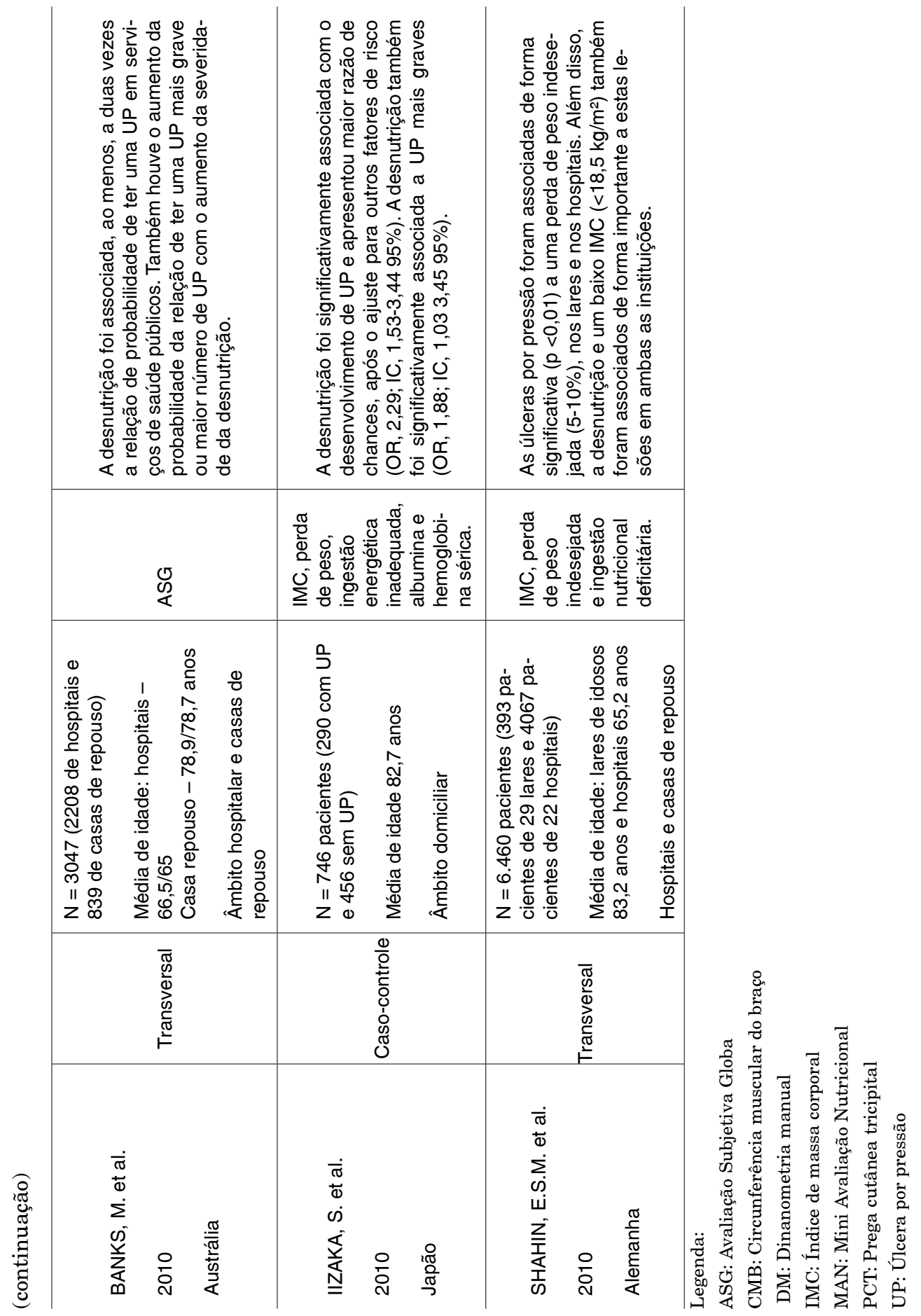




\section{Discussão}

Segundo a definição internacional da NPUAP/EPUAP (2009), uma úlcera por pressão é uma lesão localizada na pele e/ou no tecido subjacente, normalmente sobre uma proeminência óssea, em resultado da pressão ou de uma combinação entre essa e forças de torção. As UP também estão associadas a fatores contribuintes e fatores de confusão, cujo papel ainda não se encontra totalmente esclarecido. O diagnóstico do estado nutricional e do consumo alimentar dos portadores de UP é de suma importância, pois o estado nutricional interfere diretamente na reparação tecidual. A desnutrição está associada à cicatrização inadequada, por redução da produção de fibroblastos, de neoangiogênese e de síntese de colágeno, além de menor capacidade de remodelação tecidual (DESNEVES et al., 2005; COLLINS; KERSHAW; BROCKINGTON, 2005).

Segundo Projetos Diretrizes (2011), o estado imunológico alterado está associado ao risco de UP, pois a alteração da nutrição pode afetar o desenvolvimento, visto que o organismo subnutrido apresenta alteração no transporte de oxigênio e diminuição da resistência à infecção, devido a esse efeito no sistema imunológico. As deficiências de vitaminas A, $\mathrm{C}$ e $\mathrm{E}$ também podem contribuir para o desenvolvimento de UP devido ao papel que essas vitaminas desenpenham na síntese do colágeno, na imunidade e na integridade epitelial. Uma dieta rica em proteínas, antioxidantes (vitaminas A, $\mathrm{C}$ e E) e minerais, como cobre, zinco e selênio, parecem funcionar na prevenção e no tratamento das UP. A vitamina A estimula a imunidade, mantém a integridade epitelial e também a da mucosa, aumenta a síntese de colágeno e a epitelização. A deficiência de vitamina $\mathrm{C}$ está ligada ao prejuízo de cicatrização, à deiscência da ferida, à diminuição da força de tração do ferimento e à formação de colágeno defeituoso (DOLEY, 2010; HARRIS, FRASER, 2004).

A deficiência de zinco pode diminuir as taxas de fibroplastia, de epitelização e de síntese de colágeno, além de prejudicar a resposta imunológica, aumentando a suscetibilidade a infecções recorrentes (DOLEY, 2010; HARRIS, FRASER, 2004; THOMPSON, FUHRMAN, 2005). O selênio é um mineral com propriedade antioxidante, muito importante na prevenção e no controle de infecções, pois suprime a ativação de vias pró-inflamatórias, por meio da quelação de moléculas de radicais livres (VOLP et al., 2010). O cobre, por sua vez, age junto a enzimas antioxidantes e modula a ação de macrófagos e neutrófilos (TEIXEIRA et al., 2011).

Estudos apontam que a suplementação nutricional com aminoácidos (glutamina e arginina) influencia positivamente no processo de cicatrização (DOLEY, 2010; WITTE, BARBUL 2003). A glutamina pode ter alguns efeitos positivos indiretos na cicatrização de feridas, pois mantém a integridade das mucosas e diminui as taxas de infecção (DOLEY, 2010). A arginina funciona como substrato na síntese proteica, na proliferação celular, vasodilatação e na imunidade (DOLEY, 2010; YATABE et al., 2011). Assim, o adequado aporte nutricional é fundamental nas diferentes etapas de cicatrização (PROJETO DIRETRIZES, 2011). 
Desnutrição como fator de risco para úlcera por pressão

Apesar de haver diferenças nos desenhos e nos locais onde foram produzidos/publicados e de haver a presença de falhas metodológicas, tais como números reduzidos de amostra, os oito artigos utilizados para esta revisão de literatura apontam que a desnutrição é um importante fator de risco para o desenvolvimento de UP, tanto em hospitais, como em casas geriátricas ou em ambiente domiciliar. Entre os artigos utilizados neste estudo revisão, cinco foram estudos transversais, que possuem algumas limitações, por não provarem a existência de uma sequência temporal entre exposição ao fator e o subsequente desenvolvimento da doença, em que os sujeitos e suas respectivas lesões foram avaliados em um único momento.

Os estudos realizados em âmbito hospitalar de Brito, Generoso e Correia (2013), Tsutsumi et al., (2012) e Cowan et al., (2012), associaram a ocorrência maior de UP a pacientes desnutridos. $\mathrm{O}$ estudo transversal de Brito, Generoso e Correia (2013) foi realizado no Brasil nas cidades de Belo Horizonte (MG), Manaus (AM), Rio de Janeiro (RJ), São Paulo (SP), Cuiabá (MT), Natal (RN) e Curitiba (PR). Este estudo teve como objetivo determinar a prevalência de UP em pacientes internados em hospitais públicos e privados e associar UP ao estado nutricional e a outros fatores de risco. A amostra foi constituída por 473 pacientes, com uma média de idade de 58,4 anos ( $\pm 18,52$ anos). Para avaliar e classificar a severidade das UP foi utilizada a classificação das diretrizes internacionais, proposta pelo guia de prevenção e tratamento Pressure Ulcer Advisory Panel e National pressure Ulcer Advisory Panel (EPUAP/NPUAP). Para avaliar o estado nutricional dos pacientes, foram utilizadas as seguintes feramentas: avaliação subjetiva global (ASG), índice de massa corporal (IMC), prega cutânea tricipital (PCT), circunferência do braço (CB), circunferência muscular do braço (CMB) e dinanometria manual (DM). Não houve diferença significativa entre a prevalência de UP nos sete estados brasileiros estudados, indicando que esse é um problema de saúde nacional. Nesse estudo, as úlceras por pressão e a sua severidade foram diretamente associadas à desnutrição. A desnutrição apresentou associação forte e significante com a presença de UP (OR 10,46; IC 95\%: 3,25 - 33,69). Uma parcela considerável de pacientes com UP tinham CB, PCT e CMB inferior a $70 \%$ do considerado adequado $(p<0,05)$.

O estudo prospectivo realizado por Tsutsumi et al., (2012) teve como objetivo analisar a relação entre medidas antropométricas com mortalidade, UP, tempo de internação e uso de antibióticos. Os dados foram coletados no Hospital Kinen Hakuai (Tokushima, Japão), participaram do estudo 223 pacientes. Para definição do estado nutricional, foram utilizados o IMC, a PCT, a CMB e ASG. Os pacientes foram divididos em quatro grupos definidos pela concentração de albumina, PCT e CMB. O grupo A era constituído por pacientes com albumina normal $(>3,5 \mathrm{mg} / \mathrm{dl})$ e composição corporal normal (PCT e CMB > 90\% dos valores normais médios). $\mathrm{O}$ grupo $\mathrm{B}$ era 
composto por pacientes com hipoalbuminemia $(<3,5 \mathrm{mg} / \mathrm{dl})$ ou composição corporal normal (PCT e CMB > 90\%). O grupo $\mathrm{C}$ era de pacientes com albumina normal (>3,5mg/dl) e diminuição da composição corporal (PCT e CMB < 90\%). O grupo D era de pacientes com hipoalbuminemia $(<3,5 \mathrm{mg} / \mathrm{dl})$ e diminuição da composição corporal (PCT e CMB < 90\%). Nesse estudo foi observada a ocorrência de UP seis vezes maior em pacientes desnutridos do grupo D (hipoalbuminemia e diminuição da composição corporal). A mortalidade nesses pacientes foi quase dez vezes maior. Outros autores também demonstram a relação entre a hipoalbuminemia com o desenvolvimento de UP, em que a diminuição dessa substância pode causar alterações na pressão oncótica e consequentemente edema, o que compromete a difusão tissular de oxigênio e de nutrientes, predispondo a hipoxia e a morte celular (UZUN, TAN, 2007; FUOCO et al., 1997).

O estudo retrospectivo de Cowan et al., (2012) também encontrou resultados semelhantes para a relação entre desnutrição e o desenvolvimentos de UP. Esse estudo foi realizado nos Estados Unidos, e teve como objetivo investigar os riscos preditivos para o desenvolvimento de UP, por meio da análise de prontuários eletrônicos de um hospital público da Flórida, 213 prontuários foram selecionados para o estudo (100 pacientes com UP e 113 sem UP). Nesse estudo, a desnutrição foi percebida como um fator de risco para UP com um OR de 4,98 (IC $95 \%: 1,9-12,9, \mathrm{p}<0,001)$. Esse estudo também encontrou diferenças significativas entre os grupos amostrais (com UP e sem UP). O número de indivíduos com comprometimento nutricional grave e IMC abaixo de $19 \mathrm{~kg} / \mathrm{m}^{2}$ foi significativamente maior no grupo com UP. Já o número de indivíduos com IMC acima de $28 \mathrm{~kg} / \mathrm{m}^{2}$ foi significativamente mais elevado no grupo sem UP. Nesse estudo podem ter ocorrido algumas limitações, como informações incompletas nos prontuários e uma amostra reduzida.

No estudo transversal de Perrone et al. (2011) a relação do estado nutricional com UP só existiu em pacientes considerados gravemente desnutridos. O estudo de Perrone et al. (2011) foi realizado no Brasil com o objetivo de correlacionar a incidência de UP com o estado nutricional de pacientes internados em dois hospitais públicos de alta complexidade, em Cuiabá. A população do estudo consistiu de 130 pacientes com idade média de 52 anos (14-85). O estado nutricional foi avaliado utilizando a ASG. Os pacientes, posteriormente, foram divididos em desnutridos graves (ASG-C) e não desnutridos graves (ASG-A e ASG-B). A gravidade da UP foi estabelecida de acordo com a escala elaborada pela European Pressure Ulcer Advisory Panel (EPUAP). Esse estudo associou significativamente a desnutrição grave (OR 3,8 IC 95\%: 1,0$1,3 ; \mathrm{p}=0,04)$ à UP. Também apontou que a chance de ocorrer UP nos pacientes gravemente desnutridos $(20 / 49 ; 40,8 \%)$ foi aproximadamente dez vezes maior que nos pacientes considerados como não gravemente desnutridos (OR 10,4 IC 95\%: 3,6-30,5; $\mathrm{p}<0,0001$ ).

Nos estudos realizados no âmbito domiciliar por Sancho, Albiol e Mach (2012) e Iizaka et al., (2010) a desnutrição também foi relacionada à presença e ao desenvolvimento de UP. O estudo 
transversal realizado na Espanha na cidade de Tarragona por Sancho, Albiol e Mach (2012), teve como objetivo avaliar a relação entre o estado nutricional e o risco de UP em pacientes com atendimento domiciliar de dois centros de cuidados primários. Participaram desse estudo cem pacientes, com média de idade de $82 \pm 1,27$ anos. A Escala de Braden foi utilizada para avaliar o risco de UP e o estado nutricional foi avaliado utilizando o índice de massa corporal (IMC) e o questionário Mini Avaliação Nutricional (MAN). O estudo apontou que o estado nutricional afeta o risco de úlceras por pressão, com um OR 3,73 (95\% IC, $p<0,001)$ maior em pacientes desnutridos. O presente estudo demonstrou que o risco de ulceração é significativamente relacionado com o estado nutricional. A presença de UP aumentou de acordo com a piora do estado nutricional, apresentando risco de 65,2\% em pacientes em risco de desnutrição, e $85,7 \%$ em pacientes com desnutrição em atendimento domiciliar. O estudo de caso-controle realizado no Japão em 207 escritórios de atendimento domiciliar, por Iizaka et al., (2010), teve como objetivo investigar o impacto do estado nutricional e os fatores relacionados à nutrição no desenvolvimento e na gravidade das UP adquiridas no ambiente domiciliar. Participaram deste estudo 746 pacientes (290 com UP e 456 sem UP), com média de idade de $82,7 \pm 8,9$ anos. $\mathrm{O}$ estado nutricional foi avaliado utilizando o IMC, a perda de peso indesejada, a ingestão energética inadequada, a albumina e a hemoglobina séricas. Nesse estudo, desnutrição também foi significativamente associada ao desen- volvimento de UP, e apresentou maior razão de chances, após o ajuste para outros fatores de risco (OR, 2,29; IC 95\%: 1,53-3,44). A ingestão dietética adequada e a avaliação da ingestão alimentar por um profissional de saúde foram significativamente associadas à menor chance para o desenvolvimento de UP (OR, 0,43 , $0,47,95 \%$ IC, $0,27-0,68,0,28-0,79$ ).

Os estudos realizados em casas geriátricas e hospitais por Banks et al. (2010) e Shahin et al. (2010) associaram a desnutrição como um importante fator de risco de UP em ambas as instituições. O estudo transversal realizado por Banks et al. (2010), em Queensland na Austrália teve como objetivo determinar o efeito do estado nutricional na presença e na severidade da UP em indivíduos de vinte hospitais e seis casas públicas de repouso. A amostra foi constituída por duas auditorias, sendo a primeira realizada em 2002, e a segunda 12 meses depois. Na primeira auditoria, havia 774 pacientes internados em oito hospitais e 381 moradores de cinco casas geriátricas. $\mathrm{Na}$ segunda auditoria havia 1434 pacientes internados em 16 hospitais e 458 indivíduos em cinco casas geriátricas. As UP foram avaliadas por meio de definições descritas pela Australian Wound Management Association. $\mathrm{O}$ estado nutricional foi avaliado por nutricionistas treinados através da ASG. Este estudo teve como resultado que a desnutrição aumenta a probabilidade de ter uma UP em 2,6 (95\% IC, 1,8-3,5, P<0,001), para indivíduos internados em hospitais. Esse risco aumenta aproximadamente duas vezes quando o indivíduo apresenta desnutrição moderada, e quase cinco vezes quando o indivíduo apresenta desnutrição grave. 
Em casas geriátricas, o fato de apresentar desnutrição aumentou aproximadamente duas vezes a chance de ter UP em ambas as auditorias um e dois.

Shahin et al. (2010) conduziram um estudo transversal para avaliar a relação existente entre a nutrição e as úlceras por pressão adquiridas nos hospitais e lares da Alemanha. Foram avaliados 2393 indivíduos residentes em 29 lares e 4067 doentes de 22 hospitais. A Escala de Braden foi utilizada para avaliar o risco de UP e o estado nutricional foi avaliado utilizando o IMC, a perda de peso indesejada e a ingestão nutricional deficitária. As UP foram associadas de forma significativa $(\mathrm{p}<0,01)$ a uma perda de peso indesejada (5-10\%), nos lares e nos hospitais. Além disso, a desnutrição e um baixo IMC $\left(<18,5 \mathrm{~kg} / \mathrm{m}^{2}\right)$ também foram associados de forma importante a essas lesões em ambas as instituições.

Nos estudos, foram utilizados diferentes parâmetros para realizar a avaliação do estado nutricional, dos oito estudos, quatro utilizaram a ASG, que tem sido considerado um método de avaliação nutricional simples, com boa reprodutibilidade e capacidade de prever complicações relacionadas à desnutrição. Dois estudos além de outros parâmetros utilizaram a albumina sérica, as limitações na utilização desse marcador biológico, estão relacionadas a situações clínicas, como inflamação, trauma, malignidade, aumento da síntese de proteínas de fase aguda, como a proteína C-reativa (PCR), que levam à diminuição da síntese de albumina (FUHRMAN et al., 2004). Apenas um estudo utilizou somente o IMC, que apresenta algumas limitações por não indicar a composição corporal e deve ser utilizado associado a outras medidas antropométricas.

\section{Desnutrição e severidade das úlceras por pressão}

Segundo a Aspen (2007), o estado nutricional interfere na qualidade da cicatrização e influencia diretamente a relação entre anabolismo e catabolismo. A desnutrição pode influenciar a vulnerabilidade dos tecidos aos fatores extrínsecos relacionados à gênese da UP, como por exemplo, a pressão.

Além de fator de risco para o desenvolvimento das úlceras por pressão, a desnutrição também colabora para a piora dessas feridas. Dos oito artigos selecionados, dois estudos associaram a desnutrição a úlceras por pressão mais graves. Brito e colaboradores (2013) encontraram em seu estudo que o estado nutricional deficitário é considerado um risco importante na evolução de pacientes hospitalizados. Foi observado que a UP e sua severidade foram diretamente associadas à desnutrição, ou seja, $98 \%$ das pessoas que apresentaram gravidade da UP superior ou igual à fase II eram desnutridas. Esses dados corroboram o estudo de Iizaka et al (2010), nos quais a desnutrição foi significativamente associada a UP mais graves (OR, 1,88; IC 95\%: 1,03-3,45).

Outros dois estudos apontaram correlação positiva entre casos mais graves de UP em pacientes com desnutrição grave. O estudo de Perrone et al., (2011) mostrou que pacientes com desnutrição grave apresentaram significativamente 
mais lesões classificadas como graves e que cerca de $88 \%$ dos casos avançados de UP foram vistos em pacientes com desnutrição grave. O estudo de Banks et al., (2010) também demonstrou que em hospitais, ser desnutrido aumentou significativamente o risco de ter um maior número de úlceras por pressão mais graves, com o OR aumentado com a gravidade da desnutrição. Quando severamente desnutridas, o OR de ter uma ou duas UP foi de 4,2 (IC 95\%: 3,0-5,9, $\mathrm{p}<0,001$ ), aumentando ainda mais a 7,9 (IC 95\%: 3,3-18,8, p<0,001) de ter três ou mais úlceras por pressão. Esses estudos apontam que o estado nutricional tem um papel importante na gênese das UP e uma nítida relação com a sua gravidade.

\section{Excesso de peso como fator de risco ou proteção em úlcera por pressão}

O sobrepeso e a obesidade têm sido sugeridos também como fatores de risco para o surgimento dessas lesões. Segundo Pini (2012), os doentes obesos, pela dificuldade de mobilização, têm um risco acrescido de lesões teciduais precipitadas pelo posicionamento por arrastamento. Esses indivíduos apresentam sudorese intensa que provoca a maceração dos tecidos devido à acumulação da umidade nas pregas cutâneas. De outra forma, Compher et al., (2001), analisaram um estudo de coorte realizado na Filadélfia, onde foram avaliados 3214 pacientes idosos admitidos no período de 1998 a 2001. Esse estudo apontou que indivíduos com peso abaixo do normal apresentavam maiores taxas de úlceras por pressão, enquanto que indivíduos obesos apresentavam taxas menores. Os resultados sugerem que a existência de gordura corporal extra reduz o risco de desenvolvimento de úlceras em indivíduos idosos hospitalizados.

\section{Limitação do estudo}

O uso de apenas uma base de dados para a busca dos artigos pode ser considerada uma limitação do estudo. A base de dados Scopus foi escolhida por apresentar o maior número de artigos completos disponíveis sobre o assunto.

Os estudos utilizados apresentaram diferentes parâmetros para a avaliação do estado nutricional, diferentes protocolos de pesquisa e variações na qualidade metodológica. Mesmo assim, por meio dessa revisão, foi possível sintetizar evidências disponíveis na literatura e integrar com as informações sobre a relação entre a desnutrição no desenvolvimento e na gravidade das úlceras por pressão, podendo auxiliar os profissionais da área da saúde na prevenção dessa enfermidade.

\section{Conclusão}

As úlceras por pressão constituem um importante problema de saúde pública mundial e a desnutrição é um dos fatores de risco mais importantes no seu desenvolvimento e na sua severidade. Os pacientes que estão desnutridos são mais propensos a desenvolvê-la, assim, comprova-se que o estado nutricional deficitário tem um papel importante na gênese da UP e uma nítida relação com a sua gravidade. 
A avaliação nutricional é de suma importância, pois por meio dela os pacientes que apresentam risco para o desenvolvimento de UP podem ser identificados precocemente, evitando maiores transtornos e custos hospitalares. Com isso, a avaliação do estado nutricional tem papel fundamental na prevenção dessa enfermidade. Contudo, apesar das evidências encontradas nesta revisão, são necessários futuros estudos, que sejam desenvolvidos com rigidez metodológica, para reforçar tal associação.

\section{The impact of malnutrition on the development and severity of pressure ulcers: a literature review}

\section{Abstract}

The study objective was to evaluate, taking base on scientific evidences, if there exists relation between malnutrition and the risk of pressure ulcer, and the relation among the malnutrition and the seriousness of this disease. The adopted method was a literature review, using on the online Scopus database taking as reference the following descriptors: "pressure ulcer" and "nutrition". The query to de database was realized at august, 2013. Publications of the last five years, in english, spanish, and portuguese were selected. Altogether were used eight articles for this review. All the studies presented on the articles show that the risk of developing pressure ulcer is bigger in malnourished patients. Four of the studies show that is a growing probability of having severe pressure ulcer or a large number of pressure ulcers, by increasing malnutrition seriousness. Thus, it is confirmed that a deficient nutrition state has an important role in the ulcer pressure gene, and a clear relation with it gravity. The nutritional evaluation is of paramount impor- tance, because through this, the patients that have a development potential of ulcer pressure can be early identified, avoiding large nuisance and hospital costs. So, the nutritional evaluation has a fundamental role on preventing and/or treating this disease. However, based on the found evidences, future studies with a larger accuracy are necessary to reinforce this association.

Keywords: Pressure ulcers. Nutritional status. Malnutrition.

\section{Referências}

ARAÚJO, T. A.; ARAÚJO, M. F. M.; CAETANO, J. A. O uso da escala de Braden e fotografias na avaliação do risco para úlceras por pressão. Revista da Escola de Enfermagem USP, São Paulo, v. 46, n. 4, ago. 2012.

AMERICAN SOCIETY FOR PARENTERAL AND ENTERAL NUTRITION. Nutrition support core curriculum: a case based approach: the adult patient. Silver Spring (MDP): ASPEN, 2007.

BANKS, M. et al. Malnutrition and pressure ulcer risk in adults in australian health care facilities. Nutrition, Qeensland, v. 26, n. 9, p. 896-901, Sept. 2010.

BOURS G. J. et al. Prevalence, prevention, and treatment of pressure ulcers: descriptive study in 89 institutions in the Netherlands. Research in Nursing \& Health, Netherlands, v. 25, n. 2, p. 99-110, Apr. 2002.

BRITO, P. A.; GENEROSO S. D. V.; CORREIA, M. I. T. D. Prevalence of pressure ulcers in hospitals in brazil and association with nutritional status-A multicenter, cross-sectional study. Nutrition, Minas Gerais, v. 29, n. 4, p. 646-649, Apr. 2013.

COLLINS, C. E.; KERSHAW, J.; BROCKINGTON, S. Effect of nutritional supplements on wound healing in home-nursed elderly: a randomized trial. Nutrition, v. 21, p. 147-55, Feb. 2005. 
COSTA, I. G. Incidência de úlcera de pressão e fatores de risco relacionados em pacientes de um Centro de Terapia Intensiva. Dissertação de Mestrado - Escola de Enfermagem de Ribeirão Preto - Universidade de São Paulo, Ribeirão Preto, 2003.

COWAN, L. J. et al. Enhancing braden pressure ulcer risk assessment in acutely ill adult veterans. Wound Repair and Regeneration, Florida, v. 20, n. 2, p. 137-148,Mar./Apr. 2012.

CUNHA, D. F. et al. Pressure sores among malnourished necropsied adults: preliminary data. Revista do Hospital das Clínicas da Faculdade de Medicina, São Paulo, v. 5, n. 3, p. 79-82, May/June 2000.

DESNEVES, K. J. et al. Treatment with supplementary arginine, vitamin $\mathrm{C}$ and zinc in patients with pressure ulcers: a randomised controlled trial. Clinical Nutrition, Melbourne, v. 24, p. 979-987, Dec. 2005.

DOLEY, J. Nutrition Management of Pressure Ulcers. Nutrition in Clinical Practice, Baltimore, v. 25, n. 1, p. 50-60, Feb. 2010.

EUROPEAN PRESSURE ULCER ADVISORY PANEL and NATIONAL PRESSURE ULCER ADVISORY PANEL. Prevention and treatment of pressure ulcers: quick reference guide. National Pressure Ulcer Advisory Panel, Washington DC, 2009.

FUOCO, U. et al. Anaemia and serum protein alteration in patients with pressure ulcers. Spinal Cord, Rome, v. 35, p. 58-60, Jan. 1997.

FUHRMAN, M. P. et al. Hepatic proteins and nutrition assessment. Journal of the American Dietetic Association, Minnesota, v. 104, p. 1258-1264, Aug. 2004.

HARRIS, C. L.; FRASER, C. Malnutrition in the Institutionalized Elderly: the Effect on Wound Healing. Ostomy Wound Manage, King of Prussia PA, v. 50, n. 10, p. 54-63, Oct. 2004.

IIZAKA, S. et al. The impact of malnutrition and nutrition-related factors on the development and severity of pressure ulcers in older patients receiving home care. Clinical Nutrition, Japan, v. 29, n. 1, p. 47-53, Feb. 2010.

MEIJERS, J. M. M. et al. Differences in nutritional care in pressure ulcer patients whether or not using nutritional guidelines. Nutrition, Netherlands, v. 24, n. 2, p. 127132, Feb. 2008.

OLIVEIRA, M. R. M. et al. O estado nutricional na prevenção de úlcera de decúbito em pessoas acamadas. Revista Brasileira de Nutrição Clínica, Porto Alegre, v. 24, n. 4, p. 244-248, out./dez. 2009.

PERRONE, F. et al. Nutritional status and functional capacity predispose hospitalized patients to pressure ulcers. Revista de $\mathrm{Nu}$ trição, Campinas-SP, v. 24, n. 3, p. 431-438, May/June 2011.

PROJETO DIRETRIZES. Sociedade Brasileira de Nutrição Parenteral e Enteral; Associação Brasileira de Nutrologia; Sociedade Brasileira de Clínica Médica. Terapia nutricional para portadores de úlceras por pressão. 15 de julho de 2011.

SANCHO, A.; ALBIOL, R.; MACH, N. Relationship between nutritional status and the risk of having pressure ulcers in patients included in a home care program. Atencion Primaria, Spain, v. 44, n. 10, 586-594, Oct. 2012.

SERPA, L. F.; SANTOS, V. G. Desnutrição como fator de risco para o desenvolvimento de úlceras por pressão. Acta Paulista de Enfermagem, São Paulo, v. 21, n. 2, p. 367-369, Aug. 2008.

SHAHIN, E. S. M. et al. The relationship between malnutrition parameters and pressure ulcers in hospitals and nursing homes. Nutrition, Suez, v. 26, n. 9, p. 886-889, Sep. 2010.

SILVA, J. T., OLIVEIRA, M.F., SILVEIRA, M. N. Associação entre estado nutricional e incidência de úlcera por pressão em pacientes assistidos pela Equipe Multidisciplinar de Terapia Nutricional. Revista Brasileira de Nutrição Clínica, Porto Alegre, 24, n. 4, p. 217-223, June 2009. 
TEIXEIRA, E. S. et al. Avaliação do estado nutricional e do consumo alimentar de pacientes amputados e com úlceras de pressão atendidos em um Centro Hospitalar de Reabilitação. O Mundo da Saúde, São Paulo, v. 35, n. 4, p. 448-453. Ago. 2011.

THOMPSON, C.; FUHRMAN, P. Nutrients and Wound Healing: Still Searching for the Magic Bullet. Nutrition in Clinical Practice, Baltimore, v. 20, n. 3, p. 331-47, June 2005.

TSUTSUMI, R. et al. Decline in anthropometric evaluation predicts a poor prognosis in geriatric patients. Asia Pacific Journal of Clinical Nutrition, Tokushima, v. 21, n. 1, p. 44-51, Aug. 2012.

UZUN, O.; TAN, M. A prospective, descriptive pressure ulcer risk factor and prevalence study at a university hospital in Turkey. Ostomy Wound Manage, v. 53, n. 2, p. 44-56, Feb. 2007.

VOLP, A. C. P. et al. Efeitos Antioxidantes do Selênio e seu Elo com a Inflamação e Síndrome Metabólica. Revista de Nutrição, Campinas-SP, v. 23, n. 4, p. 581-590, July/ Ago. 2010.

WITTE, M. B.; BARBUL, A. Arginine Physiology and its implication for wound healing. Wound Repair Regen, Saint Louis, v. 11, 6, p. 419-423, Nov./Dec. 2003.

YATABE, J. et al. Lower Plasma Arginine in Enteral Tube-Fed Patients with Pressure Ulcer and Improved Pressure Ulcer Healing After Arginine Supplementation by Arginaid Water. The Journal of Nutrition Health and Aging, New York, v. 15, n. 4, p. 282-286, Apr. 2011. 